\title{
Living cell dry mass measurement using quantitative phase imaging with quadriwave lateral shearing interferometry: an accuracy and sensitivity discussion
}

Sherazade Aknoun

Julien Savatier

Pierre Bon

Frédéric Galland

Lamiae Abdeladim

Benoit Wattellier

Serge Monneret 


\title{
Living cell dry mass measurement using quantitative phase imaging with quadriwave lateral shearing interferometry: an accuracy and sensitivity discussion
}

\author{
Sherazade Aknoun, ${ }^{a, b, \star}$ Julien Savatier, ${ }^{a}$ Pierre Bon, ${ }^{a, \dagger}$ Frédéric Galland, ${ }^{a}$ Lamiae Abdeladim, ${ }^{a}$ Benoit Wattellier, ${ }^{b}$ \\ and Serge Monneret ${ }^{\mathrm{a}}$ \\ ${ }^{a}$ Aix-Marseille Université, Centre National de la Recherche Scientifique, Centrale Marseille, Institut Fresnel UMR 7249, 13013 Marseille, France \\ ${ }^{b}$ PHASICS S.A., Parc technologique de Saint Aubin, Route de l'Orme des Merisiers, 91190 Saint Aubin, France
}

\begin{abstract}
Single-cell dry mass measurement is used in biology to follow cell cycle, to address effects of drugs, or to investigate cell metabolism. Quantitative phase imaging technique with quadriwave lateral shearing interferometry (QWLSI) allows measuring cell dry mass. The technique is very simple to set up, as it is integrated in a camera-like instrument. It simply plugs onto a standard microscope and uses a white light illumination source. Its working principle is first explained, from image acquisition to automated segmentation algorithm and dry mass quantification. Metrology of the whole process, including its sensitivity, repeatability, reliability, sources of error, over different kinds of samples and under different experimental conditions, is developed. We show that there is no influence of magnification or spatial light coherence on dry mass measurement; effect of defocus is more critical but can be calibrated. As a consequence, QWLSI is a well-suited technique for fast, simple, and reliable cell dry mass study, especially for live cells. @ 2015 Society of Photo-Optical Instrumentation Engineers (SPIE) [DOI: 10.1117/1.JBO.20 .12.126009]
\end{abstract}

Keywords: quantitative phase imaging; lateral shearing interferometry; dry mass; living cells.

Paper 150210SSRR received Mar. 31, 2015; accepted for publication Nov. 23, 2015; published online Dec. $22,2015$.

\section{Introduction}

During the life of a cell, its dry mass, i.e., the mass of all cellular content except water, is deeply modified. These changes are related to all the metabolic and structural cell functions. The study of dry mass consequently reveals important information on cells, either as individuals or as a population. In the last decade, it has been used for yeast and mammalian cell cycle studies, ${ }^{1-3}$ to quantify biomass changes of single CD8+ T cells during antigen-specific cytotoxicity, ${ }^{4}$ or for stem cell colony studies. ${ }^{5}$ Applied to red blood cells study, dry mass measurement was proven to be a powerful tool to investigate morphological and quantitative parameters modifications during the cell cycle or diseases such as their volume, shape, or hemoglobin concentration. ${ }^{6}$

Determination of cell dry mass can be done by several methods. ${ }^{7,8}$ First, in fluorescence microscopy, fluorescent reporter protein amount is extrapolated to get the whole cell biomass, but with low precision. ${ }^{9}$ Another way is to focus on cell buoyant mass by using a suspended microchannel resonator mass sensor. ${ }^{10}$ This technique has a high precision but cannot provide individual and population data at the same time. ${ }^{11-13}$ Finally, the most common technique is quantitative phase imaging (QPI), as this approach does not imply any modification of either the sample or the substrate.

\footnotetext{
*Address all correspondence to: Sherazade Aknoun, E-mail: aknoun@ phasics.fr

†Present address: CNRS, Institut d'Optique (LP2N), UMR 5298, Bordeaux
} Université, Talence, France.
More than 60 years ago, ${ }^{14}$ the refractive index of solution was shown to be the sum of the solvent refractive index and an increment proportional to the solute mass density. If we consider the so-called projective approximation, the local optical path difference (OPD, usually expressed in millimeters) measured in the image plane of a microscope is the integral of the cell refractive index along the optical path. If the OPD is integrated over the cell surface, we obtain the so-called optical volume difference (OVD). This optical volume is proportional to the dry mass density integrated over the cell volume, which is considered to be the cell dry mass (usually expressed in picograms). We clearly see that the assumption made here is that the projective approximation is valid. This assumption shall be verified when measuring dry mass in the considered experimental conditions.

Several QPI techniques are available. Developed in the last decade, they all are label free (i.e., noninvasive) and can lead to cell dry mass measurements. We can cite digital holographic microscopy, ${ }^{1,2}$ Hilbert phase microscopy, ${ }^{15}$ Fourier phase microscopy (FPM) ${ }^{16}$ synthetic phase microscopy, ${ }^{17}$ spatial light interference microscopy, ${ }^{18,19}$ and tomographic bright field imaging based on the transport on intensity equation. ${ }^{20}$ These techniques differ on their practical implementation in biology and their accuracy. This includes, the ability to be mounted or not on any microscope, the sensitivity, the frame rate, the presence of speckle noise, the need for a reference arm, and the complexity of quantitative phase retrieval.

In this paper, we study the use of another technique based on quadriwave lateral shearing interferometry (QWLSI), using 
a commercially available wavefront sensor, the SID4Bio (PHASICS, Saint Aubin, France). ${ }^{21}$ Since this is a wavefront sensor, it is implemented like a conventional camera and is mounted in the exit port of any standard microscope.

This technique analyzes wavefront of any source type either coherent or incoherent temporally and spatially. ${ }^{22}$ Therefore, it is compatible with standard halogen or LED sources in order to get a sufficiently coherent illumination. ${ }^{21}$ Using such incoherent or low-coherence sources removes speckle noise and interference artifacts on phase images. This is a self-referenced interferometric technique that does not require a reference arm. Image is obtained in one single acquisition so fast phenomena can be studied and no artifact is generated by sample motion. It can also be simultaneously combined with other modalities such as fluorescence imaging, ${ }^{23}$ Raman, second harmonic generation, etc. To enable long time-lapse acquisitions, it is recommended to work with near infrared light, which is not absorbed by live cells. For dry mass measurements, a bandpass filter is advised to avoid OPD errors due to refractive index dispersion. The techniques was proven to reach subnanometric OPD precision on calibrated samples, with a diffraction-limited lateral resolution and a true video rate, which permits intracellular components detection ${ }^{24}$ and dynamics follow-up.

This technique already finds applications in different research domains, in biology and pure physics. However, the use of our QWLSI-based wavefront sensor for cell dry mass determination needs to be studied. To guarantee the reliability of any quantitative technique, a precise metrology study has to be performed, something that can be partially missing is some other cell dry mass studies by QPI.

In this paper, we study the metrology aspects of cell dry mass measurement process. This process includes quantitative phase measurement, cell segmentation, and dry mass assessment. All these metrological responses to these steps are individually assessed in this paper. We include measurements in real conditions of noise, resolution, sensitivity, speed, and reliability. We focus on the effect on dry mass determination of the objective magnification, its numerical aperture, and of the illumination spatial coherence. In particular, we study the dependence of dry mass measurements with focus. In the frame of the projective approximation, the dry mass is independent on the image focus. However, this is not true in practice and the dry mass measurements are reliable only over a given depth of field. We present a study of the effect of focus on dry mass determination.
This study has been done on calibrated samples. It has also been applied to mammalian cell lines. We finally present our results on dry mass of live human red blood cells, as a standard biological sample.

\section{Optical Path Difference and Dry Mass Measurement Principle}

QWLSI belongs to the family of the self-referenced wavefront sensor. This interferometric technique measures the wavefront changes introduced by the propagation of a light beam through a sample placed in the object plane of a microscope. The refractive index distribution inside the sample creates contrast in semitransparent samples like cells or tissues, using the fact that the light wavefront will be distorted when propagating through media of different refractive indices. ${ }^{21}$

A two-dimensional (2-D) diffraction grating, called modified Hartmann mask ${ }^{25}$ and placed a small distance before the sensor, is used to replicate the incident wavefront. After a small propagation, we record the interference of those replicas creates with a CCD (or CMOS, sCMOS, EMCCD) camera. The acquired interferogram is processed in real time by Fourier analysis to extract the intensity and OPD gradients. The latter are finally numerically integrated leading the OPD distribution, also called wavefront. $^{21,26}$

\subsection{Optical Path Difference}

The measured wavefront is assumed to be equal to the OPD, defined as follows:

$\operatorname{OPD}(x, y)=\int_{0}^{h}\left[n(x, y)-n_{\text {medium }}\right] \cdot \mathrm{d} z$,

where $n$ is the local sample refractive index, $n_{\text {medium }}$ is the surrounding medium refractive index, $z$ is the coordinate along the optical axis, and $h$ is the mechanical thickness of the sample.

Depending on the refractive index distribution of a sample, we can bring with QWLSI a high contrast for a semitransparent sample, compared with bright field imaging, using only an intrinsic property of light and no labeling. This can be used to deduce morphological and quantitative characteristics of cells as we will show hereafter (see Fig. 1).

The measured OPD is the OPD induced by the sample plus the OPD introduced by the imaging system which is not relevant in our case. Experimentally, in order to take into account this static OPD, we record, before beginning the measurements,

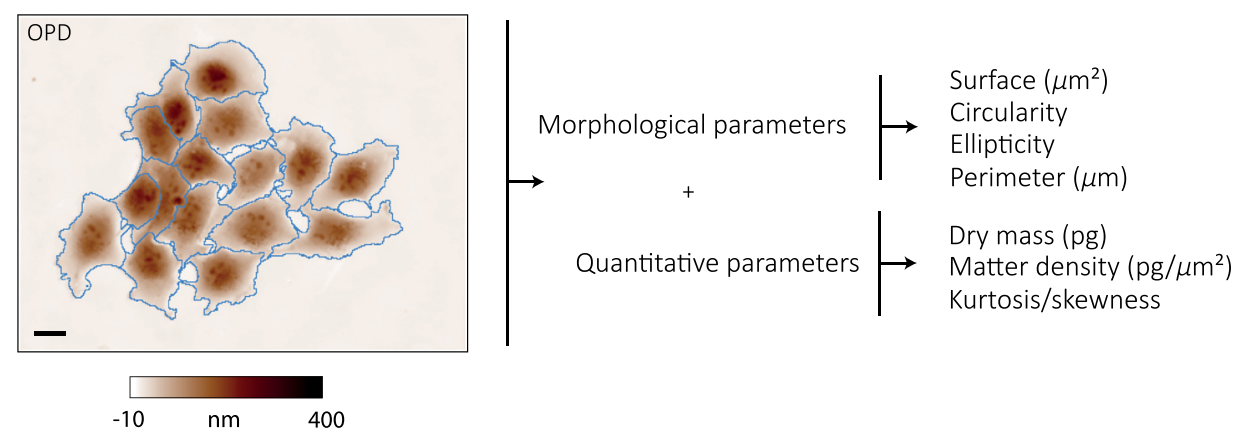

Fig. 1 Illustration of the morphological and quantitative parameters that can be extracted from an optical path difference (OPD) image obtained with quadriwave lateral shearing interferometry (QWLSI) of a sample composed by living COS-7 cells. Observation made with a $40 \times, \mathrm{NA}=0.75$ objective. The scale bar represents $10 \mathrm{~mm}$. 
a reference wavefront that will be subtracted to all subsequent OPD measurements, in an element-free region of the sample. ${ }^{23}$ This step is mandatory since microscope modifications of the wavefront are usually much larger than the cell contribution.

\subsection{Optical Volume and Specific Refractive Increment}

The OPD can be integrated over all the sample and expressed as a unit of volume. We introduce the notion of OVD, resulting from the integration of the OPD over the total projected surface of the sample and expressed in $\mu \mathrm{m}^{3}$, as follows:

$$
\mathrm{OVD}=\iint_{S} \operatorname{OPD}(x, y) \mathrm{d} x \mathrm{~d} y .
$$

Barer ${ }^{14}$ showed that we can link the phase shift introduced by a cell integrated over the sample surface to its dry mass. That can be written using our notations as follows:

$$
\iint_{S} \operatorname{OPD}(x, y) \mathrm{d} x \mathrm{~d} y=\alpha \cdot m,
$$

where $m$ is the sample dry mass and $\alpha$ is the proportionality constant called the specific refractive increment. For biological cells, the $\alpha$ coefficient lies within the range 0.18 to $0.21 \mu \mathrm{m}^{3} \mathrm{pg}^{-1}$ for proteins. Barer defined a $\alpha$ constant $=$ $0.18 \mu \mathrm{m}^{3} \mathrm{pg}^{-1}$ for most eukaryotic cells, taking into account not only proteins, but also lipids, sugars, and nucleic acids. This value is still used as a reference in this field. For particular cells like red blood cells, this coefficient is slightly different ( $\alpha=0.19 \mu \mathrm{m}^{3} \mathrm{pg}^{-1}$ ) considering that this type of cell contains a lot of hemoglobin.

\subsection{Experimental Setup}

The experimental setup used to make cell dry mass measurements is shown in Fig. 2. It is composed of a conventional inverted microscope (TE2000-U, Nikon, Japan) on which we added a bandpass filter $(\lambda=750 \pm 30 \mathrm{~nm})$ to reduce light phototoxicity during long time acquisitions on living cells. We used regular air or oil immersion Nikon objectives $(40 \times$ magnification; $\mathrm{NA}=0.75$ and $\mathrm{NA}=1.3$ and $100 \times$ magnification; $\mathrm{NA}=1.3$ ) to perform the measurements. We used the original microscope tube lens $(200 \mathrm{~mm})$ or $400 \mathrm{~mm}$ tube lens to realize additional magnification during experiments to reach optimal lateral sampling on the wavefront sensor we used. We worked with a transmission Köhler illumination which can provide an illumination numerical aperture from $\mathrm{NA} \approx 0.04$ to $\mathrm{NA}=0.52$. If not specified in the experiment, we used a numerical aperture of NA $\approx 0.08$. The QWLSI (SID4Bio, PHASICS S.A., Saint Aubin, France) was mounted on a C-mount adapter on one of the microscope's lateral camera ports or on the back video port with the $400 \mathrm{~mm}$ tube lens so that the detector plane matches the microscope image plane.

A box (Life Imaging Services, Basel, Switzerland) covering the microscope allowed us to control $\mathrm{CO}_{2}$, humidity, and temperature.

\subsection{Dry Mass Assessment Process}

In metrology, it is important to determine the measurand (here the dry mass) and the measurement process. The cell dry mass measurement process is made of several steps: acquire the OPD image, segment the cells, and then integrate the OPD over the segmented cell surface.

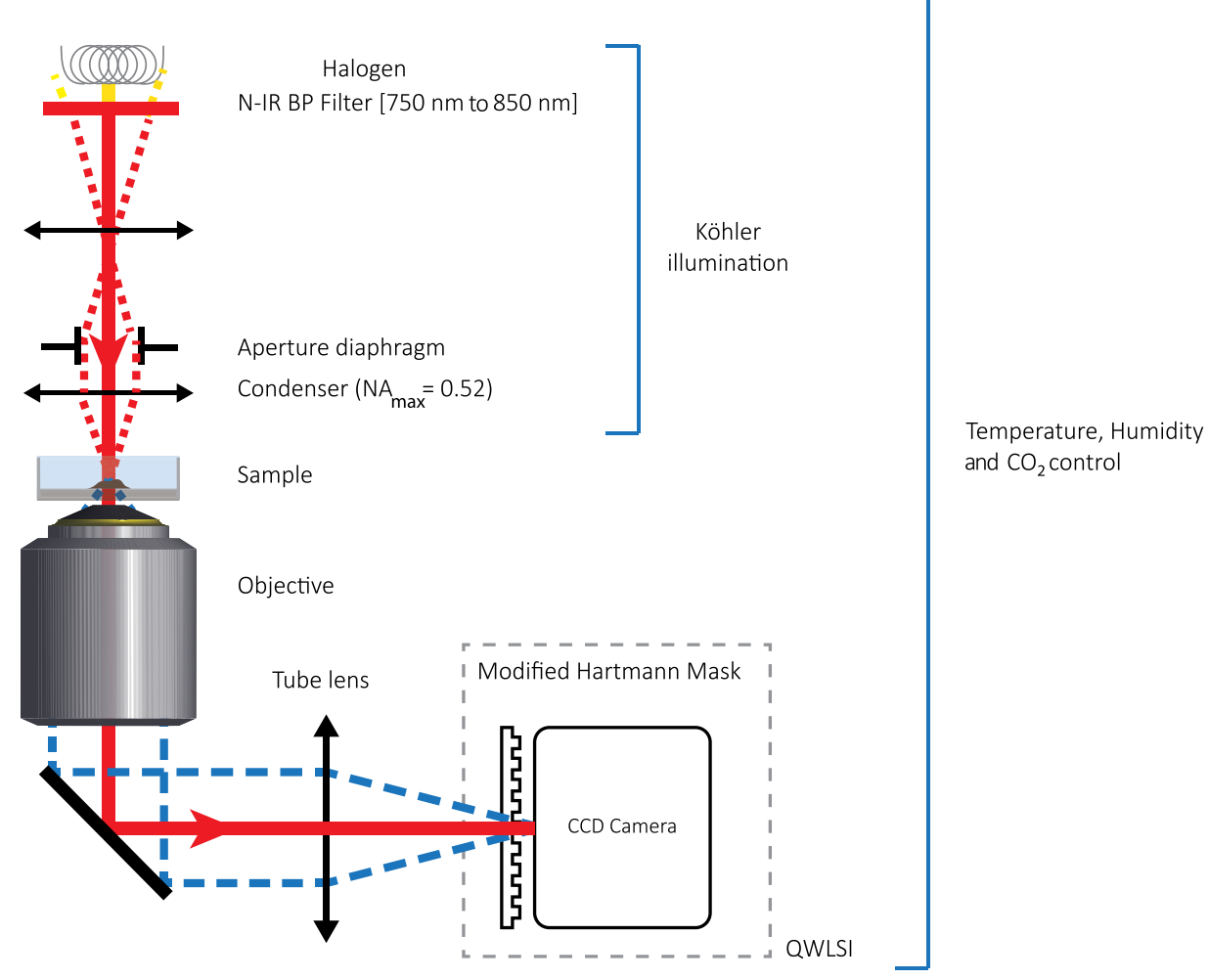

Fig. 2 Experimental setup scheme to process dry mass measurements. 
To perform dry mass measurements on cells, an image processing algorithm was developed, leading to cell segmentation from the background and individualization from other cells of the environment. This is possible thanks to the high contrast enhancement of our technique and to the total absence of artifacts (no halo around cells, no speckle, etc.). For each cell of the segmented image, the algorithm returns different morphological and quantitative measurements giving some segmented cell characteristics (see Fig. 1). The segmentation process is detailed in Appendix.

\section{Dry Mass Metrology Characterization}

In order to show the reliability of dry mass measurement, different experimental parameters of influence have been tested. In metrology, the accuracy of a method is assessed by its trueness and precision. The trueness is the difference between the average of an infinite number of measurements of the same sample and the sample reference value. Precision quantifies the dry mass value dispersion for all the measurements. The precision is usually decomposed in terms of reproducibility and repeatability. The reproducibility is the dry mass standard deviation where several factors are changed (operator, time, magnification, illumination, etc.). The repeatability is the dry mass standard deviation for independent measurements made in the same experimental conditions. Checking that the dry mass does not vary with the cell position in the field of view could be considered as a repeatability measurement. Finally, the measurement resolution, which is the smallest change detected by the measurement process, is assessed from the standard deviation of measurements made when nothing is changed except the acquisition time.

\subsection{Sources of Uncertainty}

We identified several sources of uncertainty which affect the trueness and precision of dry mass measurement. Some sources account for our means of measurement including imaging technology and data analysis and some others account for assumptions underlying the use of QPI for dry mass measurements. We study the following sources of uncertainty:

- OPD measurement noise

- segmentation errors

- field position

- cell confluence

- magnification and imaging numerical aperture

- illumination coherence

- focus

\subsubsection{Optical path difference contribution to dry mass uncertainty}

The study begins with the OPD measurement, which is the fundamental acquisition process. We evaluated the technique spatial and the temporal resolution by studying the standard deviation of OPD values of differently averaged acquisitions and successive acquisitions, respectively. A fixed sample of COS-7 cells (adherent African green monkey kidney fibroblast-like cells) was used to neglect the sample fluctuations. Cells were placed between cover slips and imaging and cell cultures were done in Dulbecco's modified Eagle medium (DMEM), supplemented with $10 \%$ fetal calf serum and sodium pyruvate. OPD images $(300 \times 400$ pixels of $0.74 \mu \mathrm{m}$ lateral size) were taken in a region free of the sample to evaluate measurement fluctuations. To measure the spatial noise, we measure the value variance of a $300 \times 400$ pixels OPD image. To get the temporal noise, for each pixel, we measure its value variance over 100 successive acquisitions. We then average the variances to lead to the average temporal standard deviation. Observations were made with a Nikon air objective $(40 \times, \mathrm{NA}=0.75)$. The results are shown in Table 1.

The measurement resolution obtained is $0.38 \mathrm{~nm}$. If the noise is white, the temporal and spatial noise should be equal. In our case, the phase is recovered from the numerical integration of phase gradients. Whereas these gradients exhibit white noise (i.e., the noise level is the same for any spatial frequency), their primitive no longer has white noise. This explains the slightly larger noise for spatial resolution. In the case where a better resolution is needed, for instance, if the object is very thin, several acquisitions are averaged to lift this limitation. By averaging 100 acquisitions, we can reach a resolution of $0.07 \mathrm{~nm} .^{27}$ This value can be compared with the $10 \mathrm{~nm}$ resolution of the spiral phase technique, ${ }^{28}$ with the $5 \mathrm{~nm}$ resolution of digital holography techniques, ${ }^{29,30}$ and with the $0.7 \mathrm{~nm}$ resolution of FPM. ${ }^{31}$

The OPD spatial resolution generates uncertainty on dry mass determination. The dry mass estimator is

$\hat{m}=\frac{1}{\alpha} \mathrm{OVD}=\frac{1}{\alpha} \sum_{i=1}^{N} \mathrm{OPD}_{i} \cdot \mathrm{pix}^{2}=\frac{1}{\alpha} S \overline{\mathrm{OPD}}$,

where pix is the pixel size in the object plane, $\overline{\mathrm{OPD}}$ is the average OPD in the cell, $S$ is the cell surface, and $N$ is the number of segmented pixels

$N=\frac{S}{\text { pix }^{2}}$.

The statistic of this estimator can be deduced from the OPD statistics, considering that the pixels are independent

Table 1 Results of spatial and temporal noise and repeatability measurements obtained on a cover slip imaged with a $40 \times, \mathrm{NA}=0.75$ objective.

\begin{tabular}{llll} 
Variable & Measurand & \multicolumn{1}{c}{ Value } & \multicolumn{1}{c}{ Measurement method } \\
\hline $\begin{array}{l}\text { Optical path } \\
\text { difference (OPD) }\end{array}$ & Spatial noise & $\begin{array}{l}0.38 \mathrm{~nm}(=0.07 \mathrm{~nm} \text { with } \\
100 \mathrm{images} \text { averaging })\end{array}$ & $\begin{array}{l}\text { OPD spatial standard deviation measurement }\left[\sigma_{\text {spatial }}(\mathrm{OPD})\right] \\
\text { on } 300 \times 400 \text { pixels images }(222 \times 296 \mu \mathrm{m})\end{array}$ \\
OPD & Temporal noise & $0.27 \mathrm{~nm}$ & $\begin{array}{l}\text { OPD temporal standard deviation measurement }\left[\sigma_{\text {temporal }}(\mathrm{OPD})\right] \\
\text { of } 100 \text { successive } 300 \times 400 \text { pixels images }\end{array}$ \\
OPD & Repeatability & $0.47 \mathrm{~nm}$ & Standard deviation of five measurements on a calibrated sample \\
\hline
\end{tabular}




$$
\begin{aligned}
\sigma_{m}^{2} & =\frac{1}{\alpha} N \cdot \operatorname{pix}^{4} \sigma_{\mathrm{OPD}}^{2} \\
\sigma_{m}^{2} & =\left(\frac{1}{\alpha}\right)^{2} S \cdot \mathrm{pix}^{2} \sigma_{\mathrm{OPD}}^{2} \\
\frac{\sigma_{m}}{m} & =\frac{\operatorname{pix} \quad \sigma_{\mathrm{OPD}}}{\sqrt{S} \quad \overline{\mathrm{OPD}}}
\end{aligned}
$$

If we consider the statistics for cell of $1 \mathrm{pixel}^{2}$, as is done sometimes in the community to qualify dry mass resolution, the spatial and temporal resolutions of the dry mass measurement will be 1.14 and $0.8 \mathrm{fg}$, respectively. This would be the measurement resolution limit if the fundamental post-treatment process, i.e., the segmentation, was not taken into account.

\subsubsection{Segmentation contribution to dry mass errors}

The segmentation process is also a source of uncertainty. This segmentation is based on an algorithm specifically designed for our technique. The main reason is that the OPD at the cell edges is usually very low. Consequently, some edge pixels could be excluded from one image to another which could alter the dry mass value. In order to estimate the performance of our segmentation algorithm in terms of induced noise on dry mass measurement, we first tested it on simulated images and then on fixed cell samples. The difficulty here is to discriminate noise coming from the OPD measurement and from the segmentation algorithm.

As OPD images always have to be segmented before extracting the parameters of interest, we first studied the segmentation error on calibrated samples. A simulation tool previously developed ${ }^{32,33}$ was used to generate OPD images of a bead $\left(n_{\text {bead }}=1.47\right.$ and $\left.n_{\text {medium }}=1.5\right)$ with varying radius going from 2.5 to $20 \mu \mathrm{m}$. The different images were segmented both automatically using the algorithm and manually to compare the results with the theoretical values. The results are shown in Table 2.

We can see that the relative error is always smaller using the proposed segmentation compared with manual image segmentation. The algorithm segmentation leads to relative errors of $<0.1 \%$. However, in general, adherent cells are more difficult to segment than beads. The main difficulty in cell segmentation is to find the plasma membrane contours, especially in lamellipodia and filopodia. Nevertheless, as these areas represent lowvalue OPD areas, even if the segmentation does not succeed in finding cell exact contours, the OPD values of the missed pixels (and so the deduced local dry mass measurement) are low and the error is negligible as it represents less than a few percent of the total cell dry mass.

The segmentation is now applied on OPD images of a living cell sample to measure the relative error of the dry mass measurement on a biological sample. We successively take 100 acquisitions of a field containing HeLa cells, mammalian cells derived from cervical cancer cells. The acquisitions are done as fast as possible to neglect natural cell mass changes: the total acquisition lasts $<50 \mathrm{~s}$. An example of dry mass fluctuations for two cells is presented in Fig. 3 and Table 3. The standard deviation on the dry mass measurement for 100 successive acquisitions is $<0.5 \mathrm{pg}$ for the two imaged cells (see Fig. 3). The relative error ( $\sigma /$ dry mass) is of $0.12 \%$ for the cell No. 1 and of $0.14 \%$ for the cell No. 2. The same kind of measurement was made on 13 cells with an averaged dry mass relative error of $0.14 \%$. Note that, in this case, the OPD noise contribution to dry mass is $24 \mathrm{fg}$ when we apply Eq. (4) with an OPD resolution of $0.4 \mathrm{~nm}$ (pixel size is $0.37 \mu \mathrm{m}$ ). Noise on OPD measurement is clearly negligible in this case.

We also applied our segmentation algorithm on images of confluent cells. We successively take 30 acquisitions of 5 Paraformaldehyde (PFA)-fixed COS-7 cells. The dry mass fluctuations are shown in Fig. 3 and Table 3. The relative error is more important in that case as compared with isolated cells. We studied 15 confluent cells leading to an average relative error on dry mass evaluation of $0.35 \%$. This is mainly due to errors while separating cells in contact with each other. Some edge pixels are assigned to either one cell or the other from one image to another.

Imaging was made with a $40 \times, \mathrm{NA}=0.75$ air objective and with a two times supplementary magnification for HeLa cells. Scale bar $=10 \mathrm{~mm}$.

\subsection{Dry Mass Measurement Reproducibility Under Different Experimental Conditions}

As the dry mass is a cell intrinsic parameter, its value for a same cell should not change with the experimental parameters such

\begin{tabular}{|c|c|c|c|c|c|}
\hline Radius $(\mu \mathrm{m})$ & $\begin{array}{c}\text { True } \\
\text { values }\left(\mu \mathrm{m}^{3}\right)\end{array}$ & $\begin{array}{c}\text { Automatic OVD } \\
\text { measurement }\left(\mu \mathrm{m}^{3}\right)\end{array}$ & $\begin{array}{l}\text { Absolute } \\
\text { error }\left(\mu \mathrm{m}^{3}\right)\end{array}$ & $\begin{array}{c}\text { Manual OVD } \\
\text { measurement }\left(\mu \mathrm{m}^{3}\right)\end{array}$ & $\begin{array}{l}\text { Absolute } \\
\text { error }\left(\mu \mathrm{m}^{3}\right)\end{array}$ \\
\hline 2.5 & 1.96 & 1.96 & $\approx 0$ & 2.51 & 0.55 \\
\hline 3 & 3.39 & 3.39 & $\approx 0$ & 4.58 & 1.19 \\
\hline 5 & 15.71 & 15.70 & 0.01 & 23.88 & 8.17 \\
\hline 7 & 43.10 & 43.07 & 0.03 & 69 & 25.9 \\
\hline 10 & 125.67 & 125.67 & $\approx 0$ & 175.94 & 50.3 \\
\hline 15 & 424.11 & 424.34 & 0.23 & 453.80 & 29.7 \\
\hline 20 & 1005.31 & 1004.72 & 0.59 & 1327 & 321.7 \\
\hline
\end{tabular}

Table 2 Comparison of optical volume difference (OVD) measurements obtained using either segmentation results obtained with the proposed algorithm or manual segmentations for OPD images of a bead of varying radius going from 2.5 to $20 \mathrm{~mm}$. 
(a)

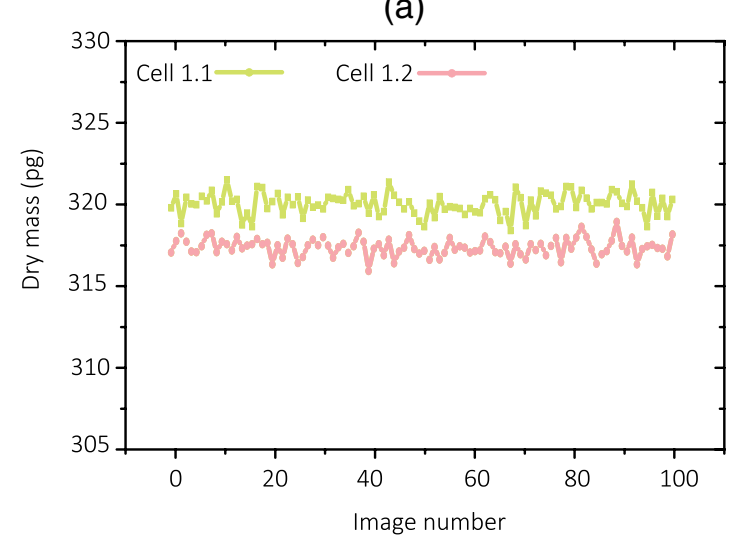

(f)

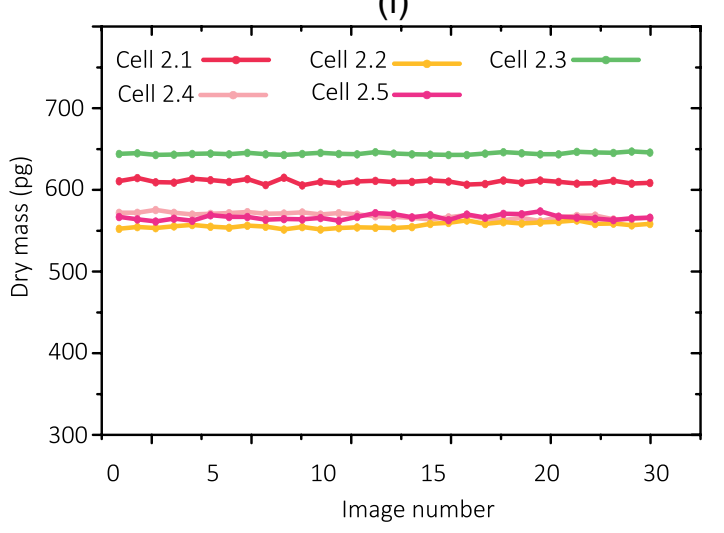

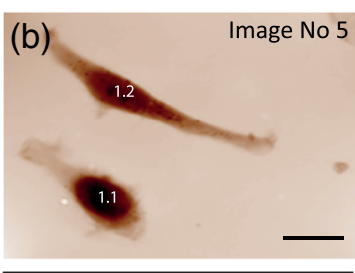
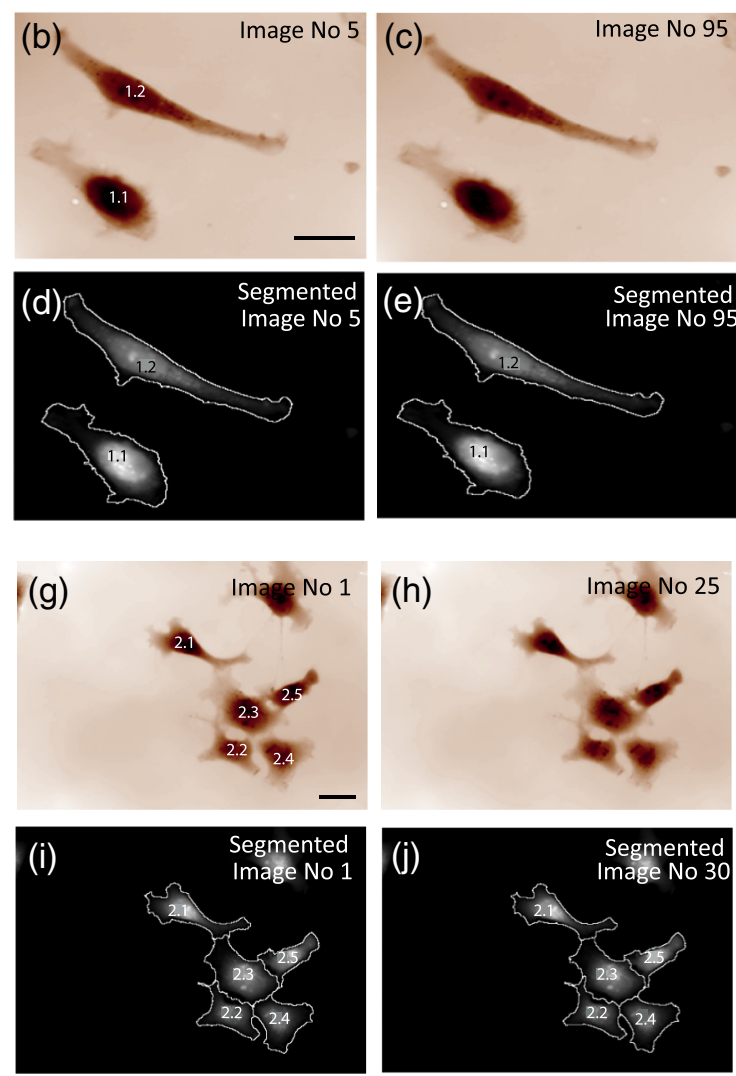
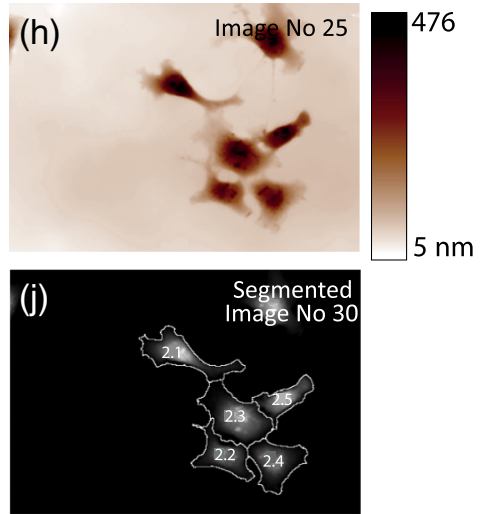

Fig. 3 Graphic representation of the dry mass fluctuations for (a) two HeLa cells and (f) five confluent COS-7 cells. (b), (c), (g), and (h) Raw OPD images. (d), (e), (i), and (j) Segmentation results of OPD images presented in (b), (c), (g), and (h).

Table 3 Results of the surface and dry mass fluctuations for the considered cells of Fig. 3.

\begin{tabular}{|c|c|c|c|c|c|c|}
\hline & & Cell 1.1 & Cell 1.2 & Cell 2.1 & Cell 2.3 & Cell 2.4 \\
\hline \multirow[t]{5}{*}{ Surface } & Average $\left(\mu \mathrm{m}^{2}\right)$ & 832.1 & 759.8 & 1573.05 & 2118.06 & 1852.37 \\
\hline & Average (pixels) & 6078 & 5550 & 2872.63 & 3867.90 & 3382.70 \\
\hline & Standard deviation $\left(\mu \mathrm{m}^{2}\right)$ & 1.6 & 2.7 & 1.67 & 3.04 & 5.29 \\
\hline & Standard deviation (pixels) & 4.2 & 7.4 & 3.05 & 5.55 & 9.67 \\
\hline & Standard deviation (\%) & 0.19 & 0.36 & 0.11 & 0.14 & 0.29 \\
\hline \multirow[t]{3}{*}{ Dry mass } & Average $(\mathrm{pg})$ & 317.74 & 319.56 & 541.35 & 610.83 & 643.39 \\
\hline & Standard deviation (pg) & 0.37 & 0.46 & 2.01 & 2.88 & 1.00 \\
\hline & Standard deviation (\%) & 0.12 & 0.14 & 0.37 & 0.47 & 0.16 \\
\hline
\end{tabular}

as magnification, numerical aperture, and illumination. In the frame of the projective assumption, it should also be independent of the focus. In practice, this is not true. The experiments presented in the following quantify the dependency of the dry mass value with the focus position.

\subsubsection{Magnification}

To quantify the dependence of dry mass measurement with magnification, experiments were done with objectives having different magnifications and numerical apertures. Each cell was measured at different positions in the field of view. The experiments were done with fixed and living cells.

Fixed cells. COS-7 cells were incubated in 2-well type II Labtek (Nunc, Denmark), in DMEM supplemented with $10 \%$ serum, $24 \mathrm{~h}$ before experiment, then fixed with 4\% PFA in Phosphate-buffered saline (PBS). They were imaged in PBS at room temperature. The microscope (Nikon Ti-U) was equipped with a turret with different classical objectives: $10 \times \mathrm{NA} 0.25$, 
$40 \times$ NA 1.3 , and $100 \times$ NA 1.3 . Cells were imaged at 25 positions twice [see Figs. 4(a) and 4(b)] with these three objectives. Köhler and exposure time adjustment were done for each objective as well as the reference image. Aperture diaphragm was closed at $10 \times$, opened at $5 \%$ at $40 \times$ and at $10 \%$ at $100 \times$. No averaging was done during image acquisition to mimic live cells experiment, where we try to get images as fast as we can, avoiding intracellular motion blurring.

The dry mass measurement results are represented with bee swarm plots [Fig. 4(c)]. The median value appears to be comparable for each presented case with a standard deviation of $<7$ pg (relative error $\sigma /$ median $<1 \%$ ). As we have a limited number of samples, a nonparametric test ( $U$ Mann and Whitney) ${ }^{34}$ was performed to compare the different cases (BD versus $\mathrm{SD}$ ) for each magnification and the three different magnification between them. This test showed that the differences between the different tested populations are not significant.

Identical measurements were done on 15 COS-7 cells. We chose to present graphically the results for three of them showing the relative difference to the median value (see Fig. 5). The results are also summarized in Table 4 where we show the averaged relative error on the 25 positions for each objective.

We can see that the measurement dispersion is higher at lower magnifications. One reason could be that the cell size in pixels is smaller, which enhances segmentation errors. Observing the images, we see that the main reason is that out-of-focus objects become visible at low magnification and alter the OPD, and thus the dry mass. The reason is that at low magnification and NA, depth of field is much larger than at high NA. Therefore, debris or dust, at the different interfaces of the well, modifies the OPD values. We decided not to exclude such cases where the artifacts were obvious because in automated situations it would be impossible to detect and remove them in real time. We also see that dispersion on dry mass values is smaller for small displacements, even more at high magnification (Table 4). This is mainly due to differences in the segmentation result.

Living cells experiment. COS-7 cells were incubated in $\mu$-Slide 2 well $\mathrm{Ph}+$ chambers, in DMEM supplemented with $10 \%$ serum, $24 \mathrm{~h}$ before experiment. They were imaged in HBSS + HEPES, at $37^{\circ} \mathrm{C}$. Two independent experiments were done, one with $10 \times$ NA 0.25 objective, one with $40 \times$ NA 1.3 (used as $60 \times$ with $\times 1.5$ tube lens). For each experiment, white light was filtered in near infrared with a LP750 nm filter, to avoid cell damages. No averaging was done on image acquisition. Figure 6 shows (a) surface and (b) dry mass of 17 live cells, imaged in five positions (a smaller number of positions was considered here as we were imaging live cells) with $10 \times$ NA 0.25 objective. Surface and dry mass are not strictly correlated, as COS-7 cells can reach a high range of surface for a given dry mass, mostly due to their motility capabilities. When they enter mitosis, their surface will also decrease. The mean standard deviation for the five positions for the surface is $(9.4 \pm 3.3) \%$ and for the dry mass $(7.1 \pm 2.3) \%$. Figure 7 shows dry mass and surface of nine other live COS-7 cells,

(a)

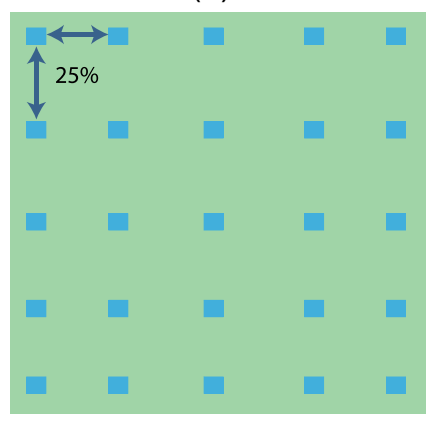

(b)

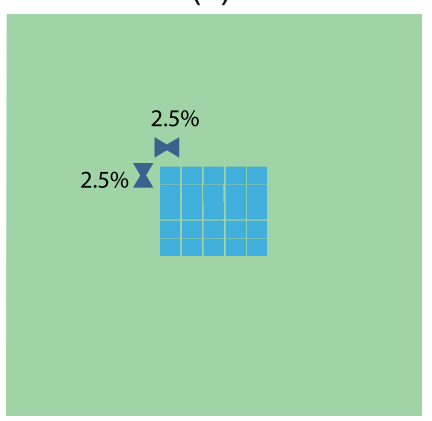

(c)

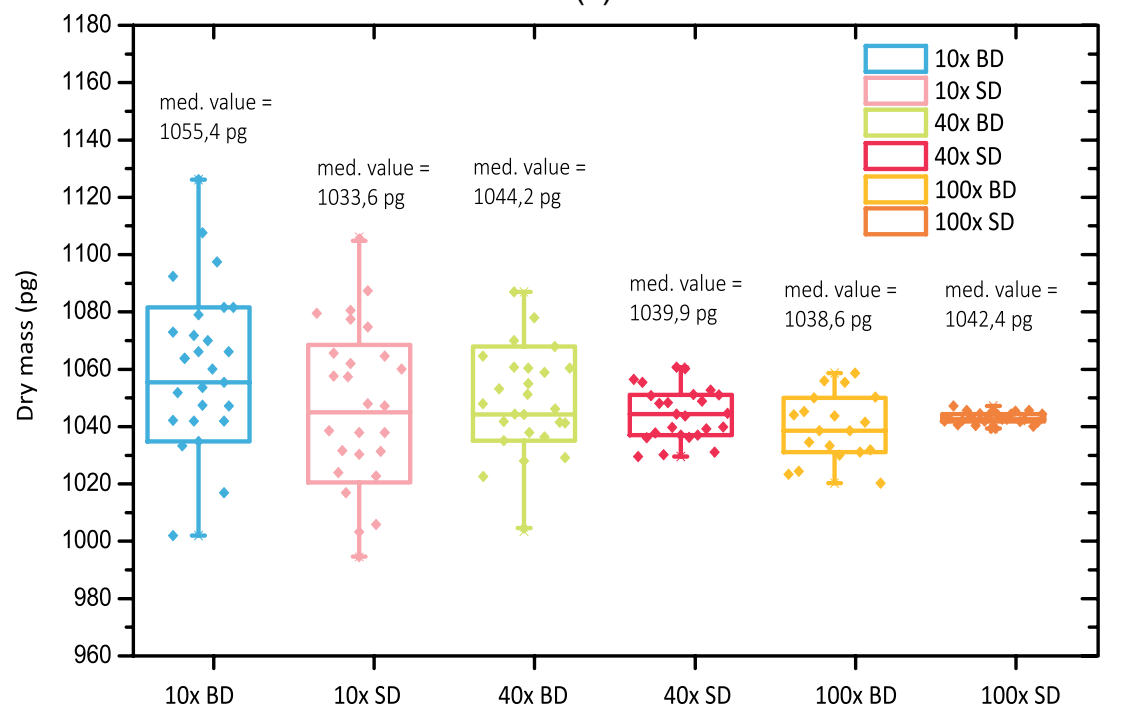

Fig. 4 (a, b) Schematic representations of the different studied sample positions. One blue square represents one position. Two different experiments were made by moving the sample 25 times in the field of (a) $25 \%$ and (b) $2.5 \%$. Bee swarm representation of dry mass measurements for one fixed COS-7 cell, obtained with three objectives: $10 \times$ NA $0.25 ; 40 \times$ NA 0.75 ; and 100 NA 1.3, in this order (c). Statistical parameters such as median, quartiles, minimum, and maximum values are graphically represented. med. is the median, BD is the big displacement corresponding to case (a) where $100 \%$ of the field is scanned and $S D$ is the small displacement corresponding to case $(b)$ where only a small part of the field $(\approx 12 \%)$ is scanned. 


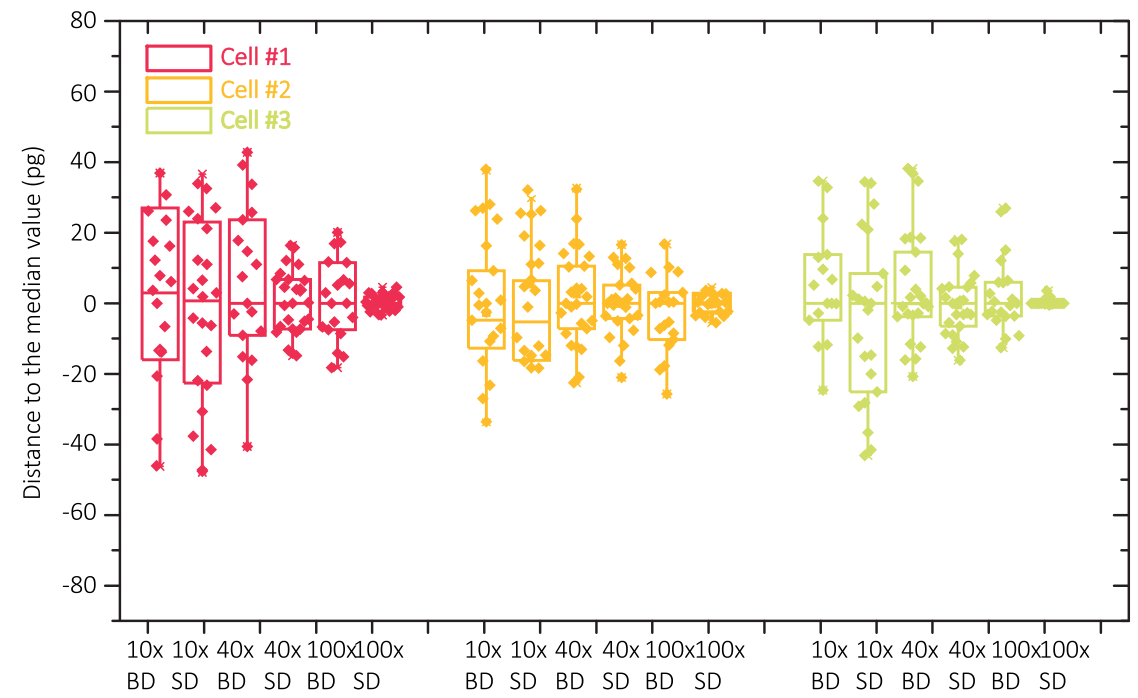

Fig. 5 Bee swarm representations of dry mass measurements of three cells with different sample positions [Figs. 4(a) and 4(b)]. Two different experiments were made by moving the sample in the field. Bee swarm representation of dry mass measurements for one fixed COS-7 cell, obtained with three objectives: $10 \times \mathrm{NA} 0.25 ; 40 \times \mathrm{NA} 0.75$; and $100 \times \mathrm{NA} 1.3$, in this order (c). BD is the big displacement and SD is the small displacement.

Table 4 Averaged relative error on dry mass measurements obtained on 15 fixed COS-7 cells. Each cell was imaged with the three different objectives previously described with multiple positions in the field.

\begin{tabular}{lccccc}
$\begin{array}{l}\text { Relative error } \\
10 \times \text { BD }(\mathrm{nm})\end{array}$ & $\begin{array}{c}\text { Relative error } \\
10 \times \mathrm{SD}(\mathrm{nm})\end{array}$ & $\begin{array}{c}\text { Relative error } \\
40 \times \mathrm{BD}(\mathrm{nm})\end{array}$ & $\begin{array}{c}\text { Relative error } \\
40 \times \mathrm{SD}(\mathrm{nm})\end{array}$ & $\begin{array}{c}\text { Relative error } \\
100 \times \mathrm{BD}(\mathrm{nm})\end{array}$ & $\begin{array}{c}\text { Relative error } \\
100 \times \text { SD }(\mathrm{nm})\end{array}$ \\
\hline $4.37 \%$ & $3.87 \%$ & $2.42 \%$ & $1.22 \%$ & $0.98 \%$ & $0.28 \%$ \\
\hline
\end{tabular}

imaged in five positions with $40 \times$ NA 1.3 objective (used as $60 \times)$. The mean standard deviation for the surface is $(2 \pm 1.6) \%$, and for the dry mass $(1.8 \pm 0.5) \%$. As seen for fixed cell, we notice that the dry mass dispersion is larger at low magnification than at large magnification.

\subsubsection{Spatial light coherence}

Dry mass measurement from OPD images implies that the sample should be illuminated with plane waves. To make this measurement simple and not experimentally invasive, we use a Köhler illumination, commonly installed in any microscope and not a laser source. However, we need to check that dry mass measurement is not altered by a spatial partial incoherence. In this section, we show that its influence on dry mass measurements is weak in case of small numerical aperture illuminations. The results are shown in Fig. 8. OPD measurements were first done by imaging fixed HeLa cells with different aperture diaphragm apertures. The actual aperture size was controlled with calibrated objects going from NA $=0.08$ to the highest aperture of 0.22 corresponding to half of the maximum aperture of the diaphragm.

The relative error measurement is $<0.7 \%$ with a dry mass variation between the two extreme points of $0.64 \mathrm{pg}$, proving that the measurement is not dependent of light spatial coherence, i.e., not user dependent.

The impact of spatial light coherence was also studied on calibrated samples. Microparticles based on polystyrene, with $n=\sim 1.59$ (not specified by the manufacturer), $30 \pm 0.4-\mu \mathrm{m}$ diameter (Fluka, Sigma-Aldrich, Lyon, France) were diluted 100 times, dried on a coverslip and embedded in 1.544 refractive index immersion oil to be close to a mitotic mammalian cell, either in size and OPD. White light was filtered in red with $700 / 70 \mathrm{~nm}$ band pass filter to be able to compare our results with tabulated refractive indices at this wavelength. Dry mass has no physical significance in this case. We will only talk about OVD. Three beads were imaged with a $100 \times$ NA 1.3 objective and $0.52 \mathrm{NA}$ condenser. Aperture diaphragm was set at $5 \%, 25 \%, 50 \%, 75 \%$, and $100 \%$ of full illumination aperture. This percentage corresponds to the position of the diaphragm manual stick. Five percent corresponds to a minimal standard value for coherent spatial illumination, keeping enough photons on the wavefront sensor for short acquisition time $(<500 \mathrm{~m} / \mathrm{s})$. The focus was made at the equatorial plane and set constant for the different apertures. A reference was taken for each condition of illumination, for each bead. We used our regular algorithm to determine the OVD of the bead.

Figure 9 shows the influence of the aperture diaphragm aperture on OVD. It is not constant all over the full aperture. However, the variations represent $<1 \%$ of OVD at $25 \%$ and $50 \%$, and $<2 \%$ at $75 \%$ and $100 \%$ opening (with respect to $5 \%$ opening). This is lower than variabilities seen on biological samples. The variation is also not linear. OVD increases between $5 \%$ and $25 \%$ opening, then decreases till $75 \%$, and is stable till $100 \%$. The decrease at high aperture is explained from our previous studies where we showed that quantitative values are spatially filtered by the imaging process. ${ }^{22}$ The quantitative values are also altered at low apertures because of diffraction 
(a)

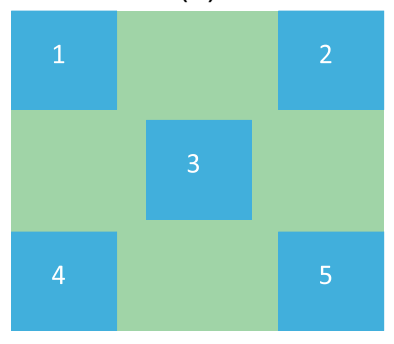

(b)

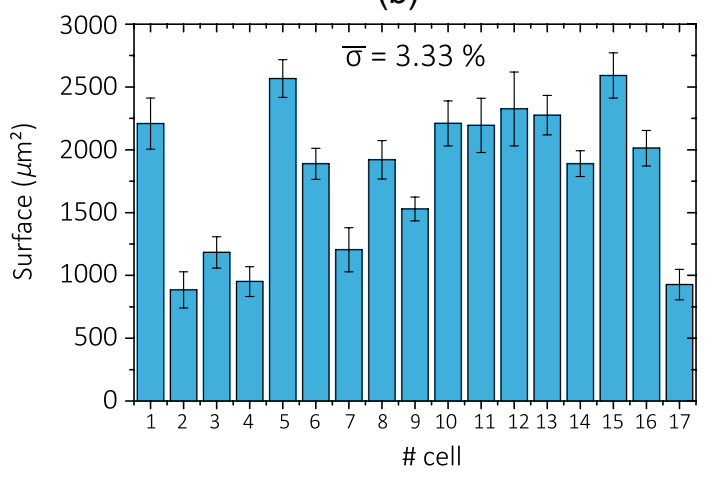

(c)

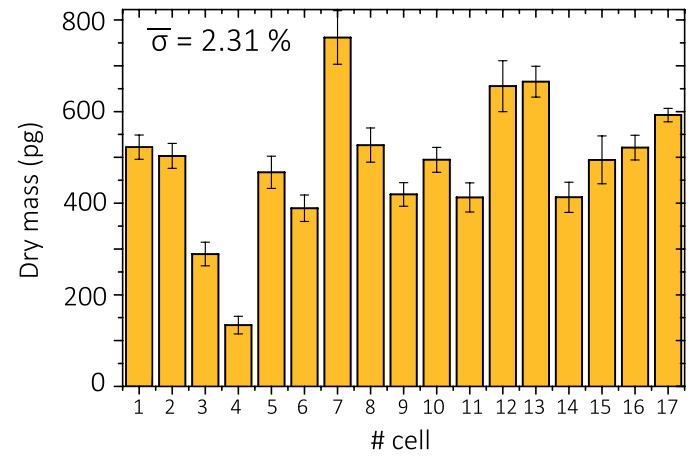

Fig. 6 (a) Schematic representations of the different studied sample positions. Bar diagrams of (b) surface and (c) dry mass of 17 live COS-7 cells, respectively, taken with 10× NA 0.25 objective. Error bars: standard deviation over five positions in the image.

rings around the bead sharp edges appearing at low-coherence illumination. In conclusion, illumination spatial coherence has little influence (inf $2 \%$ for any illumination; inf $1 \%$ for illumination close to the minimal aperture) on dry mass measurements, making the measurements insensitive to operator setting. Using a standard Köhler illumination is therefore possible, though it is recommended to keep the aperture diaphragm close to closure.

\subsubsection{Focus}

In dry mass studies, it is assumed that the dry mass is integrated from OPD measurements. OPD measurements themselves are considered as the integral of the refractive index along the axial direction over the sample thickness. This assumption neglects refraction and diffraction. By definition, the OPD is then independent on the focus position. This means that the dry mass should not depend on image focusing. However, this is experimentally not verified because diffraction effects induce a depth of field effect which attenuates the contribution of the out-of-focus planes far from the focus position. The OPD value is then altered and its value depends on the focus position. To keep the dry mass measurement accurate, it is necessary to determine a focus range where the dry mass value is reliable. On a living COS-7 cell sample placed in their culture medium, a stack of OPD images is realized on a mitotic cell of $40-\mu \mathrm{m}$ diameter by taking one image in one plane and translating the objective along the optical axis between -10 and $+10 \mu \mathrm{m}$ from (a)

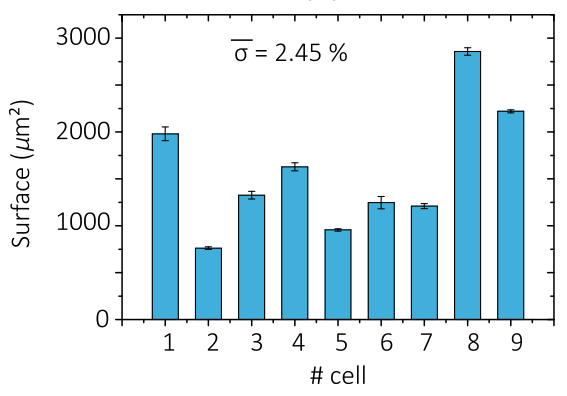

(b)

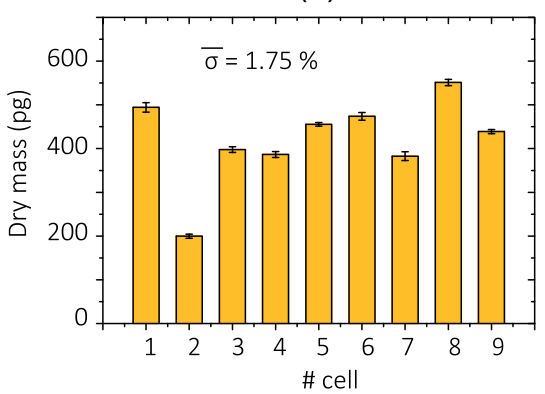

Fig. 7 Bar diagrams of (a) surface and (b) dry mass measurements for 9 COS-7 cells, respectively. The error bars were calculated by evaluating the standard deviation value of the measurement taken in five different field positions. Imaging was done with $40 \times$ immersion oil objective $(N A=1.3)$ with a supplementary magnification of $1.5 \times$. 


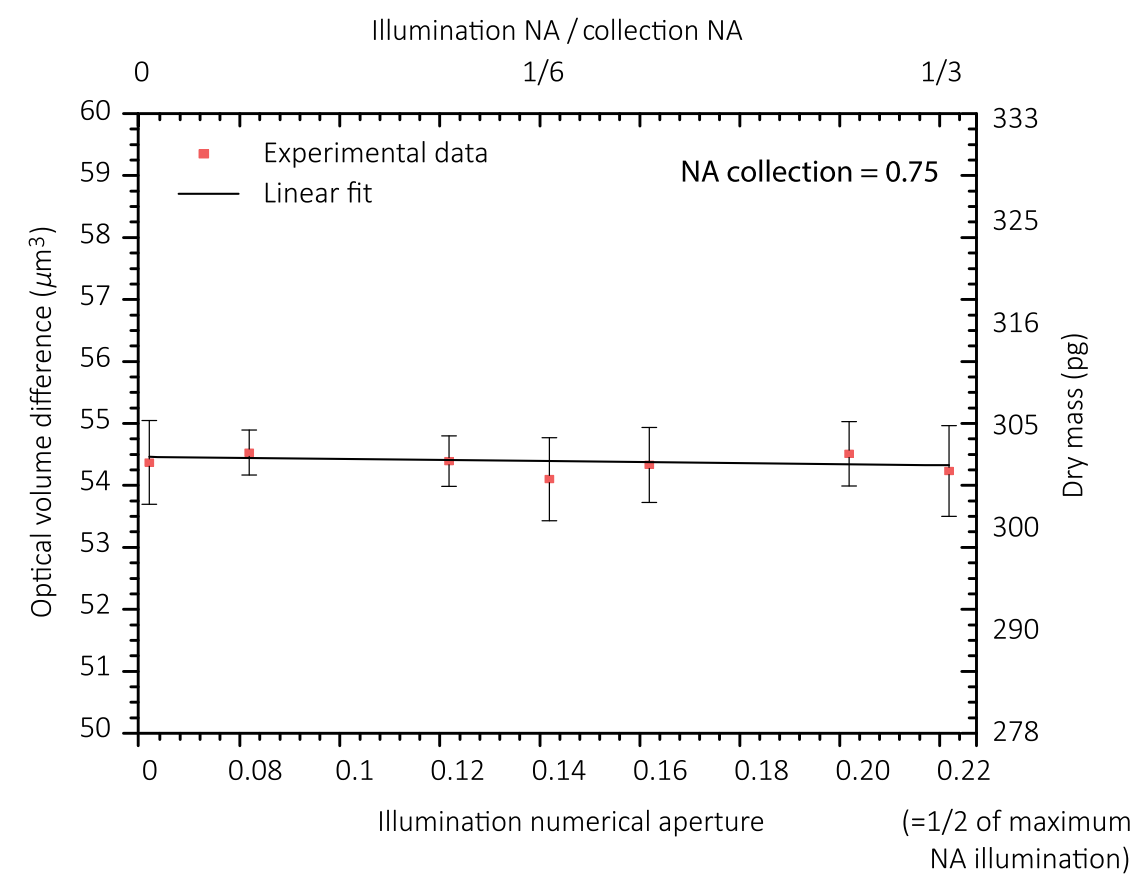

Fig. 8 OPD evolution with the spatial coherence on a fixed cell sample. Observations made with $40 \times$, $\mathrm{NA}=0.75$ objective, and conventional $0.52 \mathrm{NA}$ condenser. The error bars were calculated by evaluating the standard deviation value of a $50 \times 50$ pixel area of the image.

the focus plane. The same experiment is done on an adherent COS-7 cell. The results are shown in Fig. 10.

We can see that the measurement is dependent on the focus. The OVD has a parabolic variation being maximum close to the image plane. This can be used to determine the cell focus position with a $z$-stack, as the focal plane corresponds to an OPD maximum value. It can be interesting to note that the focal plane here corresponds also to an intensity contrast minimum. We see that this focus dependence is stronger for the mitotic cell with a dry mass value varying fast when defocusing.

We can define two focus ranges, depending on the studied sample, where the dry mass does not vary $>1 \%$ of its value at the focus position. For the mitotic cell, we can make images from $\pm 2 \mu \mathrm{m}$ for the focus position and from $\pm 5 \mu \mathrm{m}$ for the adherent cell.

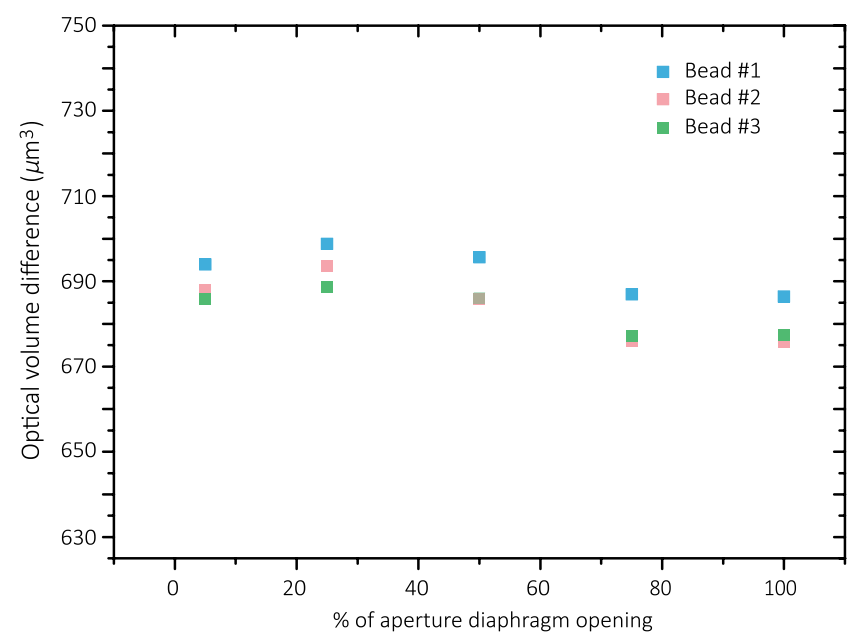

Fig. 9 Influence of aperture diaphragm aperture on optical volume difference (OVD) of $30 \mu \mathrm{m}$ polystyrene beads.
We can see that the dry mass measurement dispersion is bigger for the mitotic cell. This is mainly due to segmentation errors.

We did the same kind of experiment on calibrated samples, in the same conditions than at Sec. 3.2.2. Three beads were imaged with a $40 \times$ NA 1.3 objective (used as $60 \times$ with $1.5 \times$ tube lens). $z$-stack series of images were taken to observe the influence of focusing on a calibrated object. Twenty-nine images were taken (14 above and 14 below the equatorial plane determined on intensity image) every $500 \mathrm{~nm}$, using PI nano $X Y Z$ controller (PI, Karlsruhe, Germany). A single reference was taken at the equatorial plane focus, in an empty area. On each image, we used our regular algorithm to determine the OVD. Figure 11

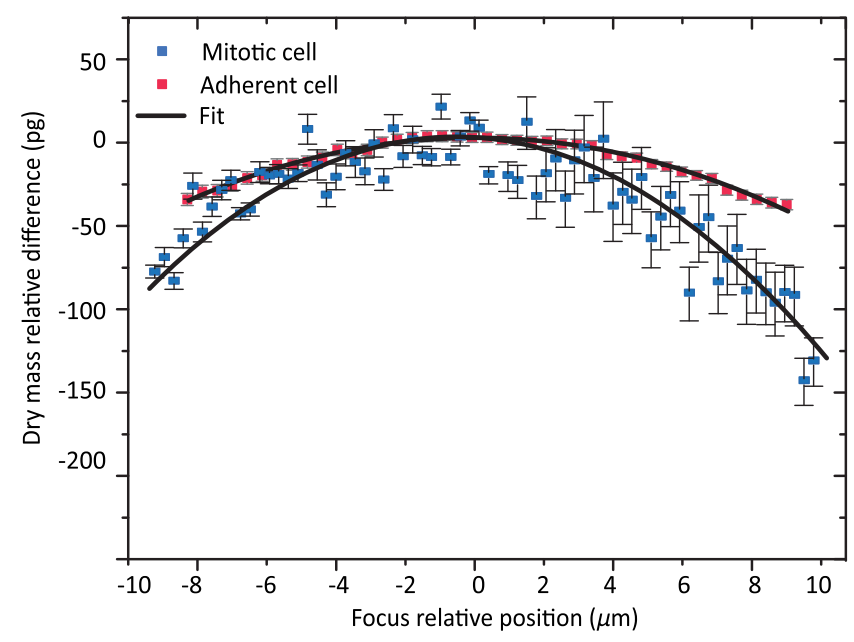

Fig. 10 Results of the relative dry mass evolution with the focus relative position of a mitotic $40-\mu \mathrm{m}$ diameter and an adherent COS-7 cell. The error bars were calculated by evaluating the standard deviation value of a $50 \times 50$ pixel area of the image. 


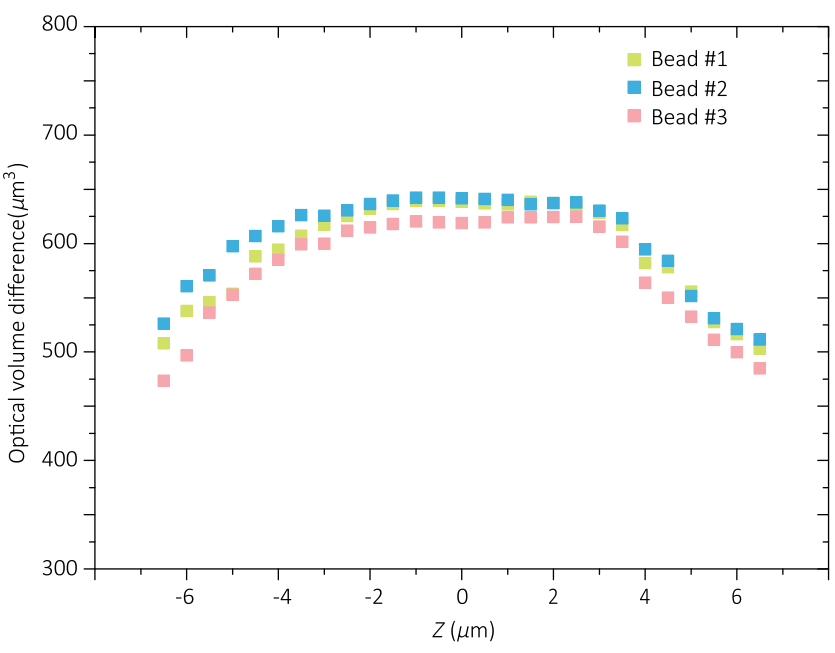

Fig. 11 OVD variation from both sides of the focus position. Three polystyrene beads imaged with a $40 \times, \mathrm{NA}=1.3$ objective.

shows the OVD at different $Z$ positions, with respect to equatorial plane $(0 \mu \mathrm{m})$, visually determined on intensity image. We see that OVD reaches a plateau at $\pm 2 \mu \mathrm{m}$ around the 0 position. At $+2.5 \mu \mathrm{m}$, the OVD rises up a bit, then decreases, faster than it increases before the plateau. All over the plateau, OVD has a variability always lower than $1 \%$ compared with the OVD at $Z=0$, for each bead (mean variability at the plateau: $0.27 \%$, $0.37 \%$, and $0.43 \%$ for beads 1 to 3 , respectively). At $Z=0$, for the first bead, $\mathrm{OVD}=638.45 \mu \mathrm{m}^{3}$; and diameter is
$30.2 \mu \mathrm{m}$, which gives a $d n=0,046$; and $n_{\text {bead }}=1.59$, which is in good agreement with the literature.

This proves that it is important to keep the cell in focus to accurately and reproducibly measure their dry mass. Note that since QWLSI recovers the complete scalar electromagnetic field, the latter can be numerically propagated in different planes in order to compensate for any focus drift during a large sample scan. $^{35}$

\section{Red Blood Cell Application}

The measurement was applied to red blood cell dry mass measurements. These cells are used in QPI as a model because of their low variability. Indeed, one can easily compare the results obtained to the mean values that can be found in the literature with different techniques. Human blood sample from healthy patient was diluted into PBS to a $0.1 \%$ final concentration. It was incubated with DNA-labeling fluorescent dye Hoechst 33342 to discriminate red blood cells from other blood cells, which contain DNA, then washed and put into PBS. Cells were allowed to settle in a 2-well type II Labtek (Nunc, Denmark) few minutes prior to measuring. Imaging by QWLSI was done at room temperature with a total $200 \times$ magnification with a $100 \times 1.3$ NA objective to obtain a good sampling on the sensor as red blood cells diameter is of few micrometers. An example is shown in Fig. 12. We can see an OPD image of a red blood cell [Fig. 12(a)] and with high pass filtering to remove low frequencies [see Fig. 12(b)]. The recognizable biconcave shape can be highlighted thanks to a profile plot drawn in Fig. 12(a). In order to measure the sensitivity of our measurement, the spatial (a)

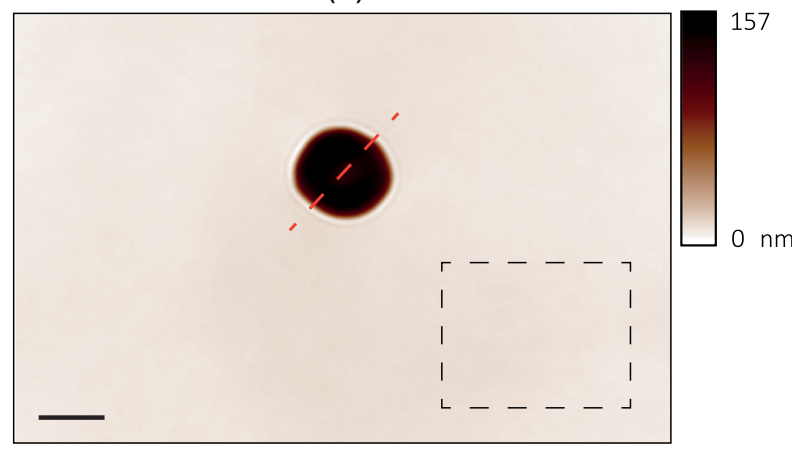

(c)

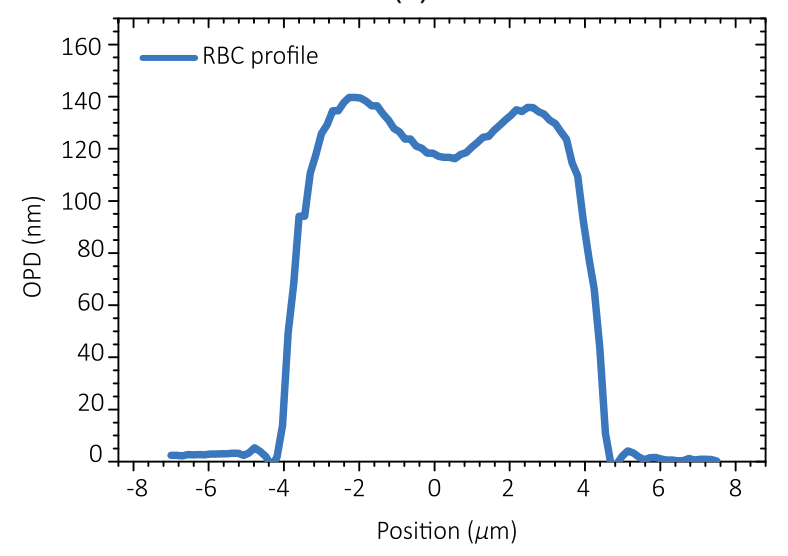

(b)

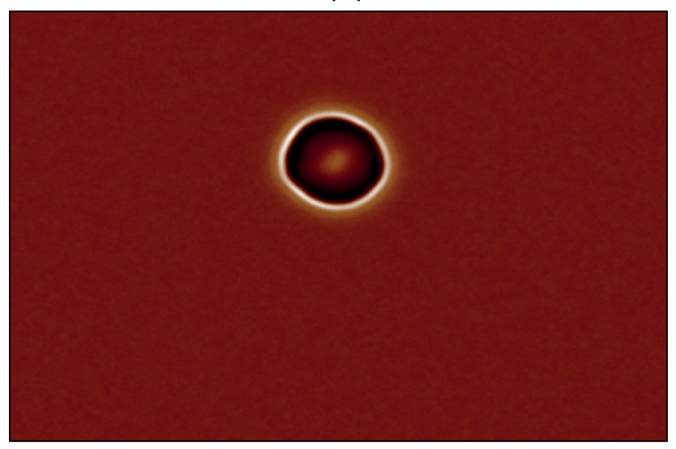

(d)

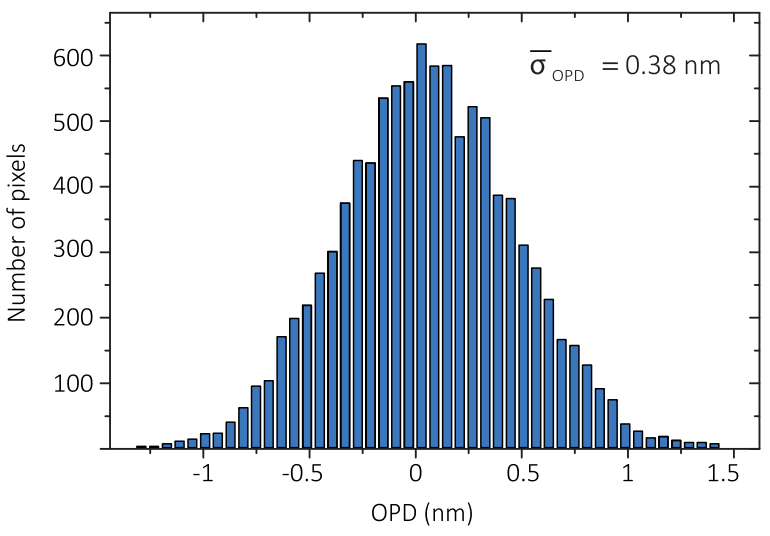

Fig. 12 (a) Raw and (b) high pass filtered OPD images of a single red blood cell. (c) Profile plot drawn on (a) following the red dotted line. (d) OPD distribution of the area plotted on (a) in black dotted lines. 


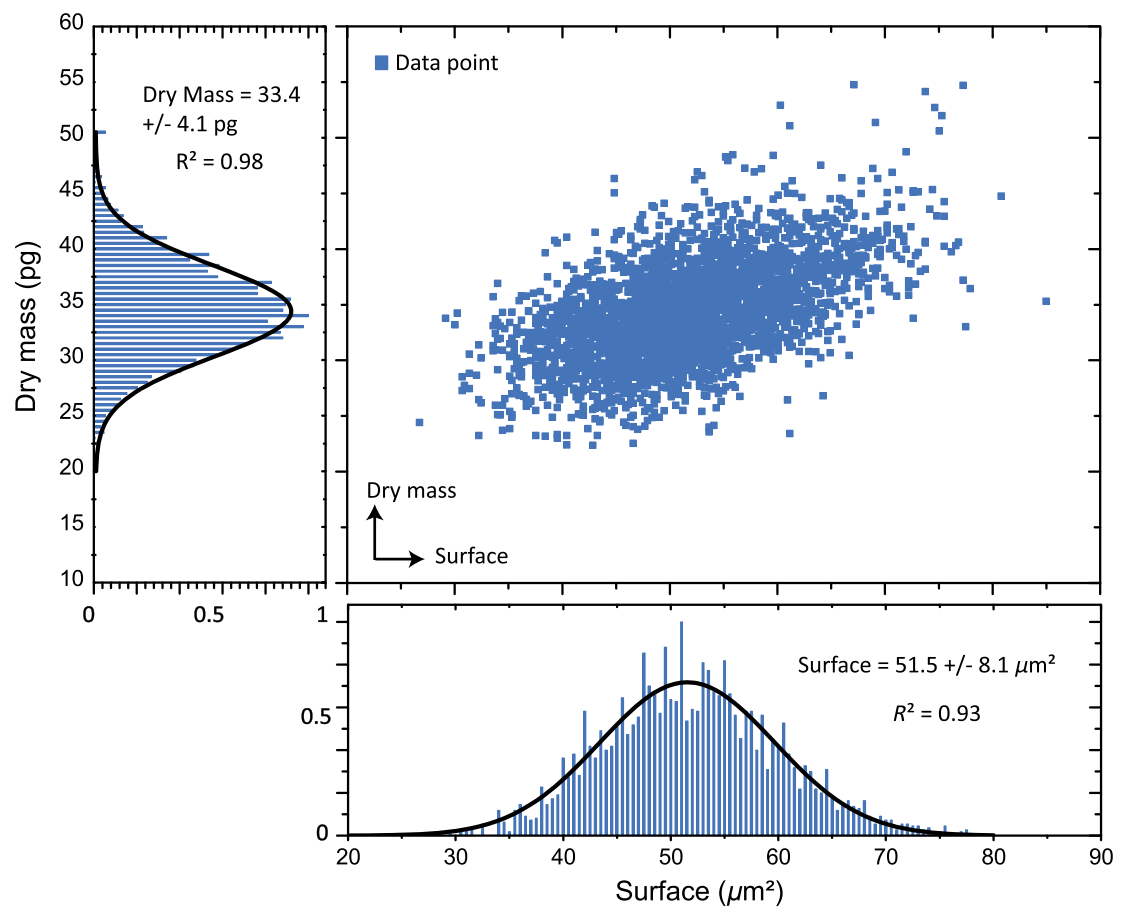

Fig. 13 Red blood cell dry mass function of the surface and population repartitions in function of the dry mass and the surface, respectively. Statistics were made on a population composed by 3220 red blood cells and imaging with a $63 \times$ magnification.

standard deviation value on an OPD image was calculated on a $50 \times 50$ pixel area [area in dotted lines in Fig. 12(a)] and leads to a mean value of $0.38 \mathrm{~nm}$.

A population study was realized on 3220 red blood cells. The results are shown in Fig. 13. The presented histograms show the population repartition in terms of dry mass and surface. We can see that the histograms have a Gaussian shape (see $R^{2}$ values of the histograms Gaussian fitting) and are centered around a value of $33.4 \mathrm{pg}$ for the dry mass and $51.5 \mu \mathrm{m}^{2}$ for the projected surface. The standard deviation value of the dry mass is of $4.1 \mathrm{pg}$ showing the low variability of the studied cellular population. This value is in agreement with results found in the literature $\left(31.4,{ }^{14} 27\right.$ to $32,{ }^{36}$ and $\left.27.3 \pm 5.3 \mathrm{pg}^{37}\right)$.

\section{Conclusion}

In this paper, we have presented the measurement of biological cell dry mass from OPD measurement with a QWLSI wavefront sensor. Cell dry mass study with this process is easy to do and reliable. The dry mass measurement has been characterized in terms of repeatability and reproducibility showing the influence of experimental parameters on the final result. We analyzed different sources of variability or error.

We showed that the method is insensitive to magnification and spatial light coherence. However, at low magnification any debris, scratch or defects in the extracellular medium, slide, dish, or coverslip alters the dry mass value because the depth of field is larger than at high magnification. This point is very important in the case where automated acquisition and analysis is made on a large population, where manual correction would take too much time to eliminate suspicious cells.

We studied the repeatability of dry mass measurement at different camera field points. In general, the value dispersion is around $1 \%$. At low magnification $(10 \times)$, the dry mass variability is higher and at higher ones (40 and even more 60 and $100 \times$ ) it is lower. It is a question of number of pixels per cell but also of the difficulty to have really clean background. With low magnification comes high field depth, where any dirt on the other side of the coverslip, or even on the coverslip above the medium like in the $\mathrm{Ph}+\mu$-Slide, has influence on the measured OPD. When we subtract background with our segmentation algorithm, we take into account low spatial frequencies, but not high ones, what could lead to errors. The variability at low magnification is a bit better with live than with PFA-fixed cells. It can be due to the effect of fixating agent on the cell, especially on the integrity of the plasma membrane and on the three-dimensional (3-D) architecture of the cytoskeleton. Further investigation should be done on the effects of fixation on dry mass. At high magnification, the position in the field of view has no significant effect on dry mass measurement.

The effect of focus has also been analyzed. On an adherent flat cell, focus is easy to do and is never wrong more than a few hundreds of nanometers. On a thicker and more spherical sample, like a mitotic cell or a 30-mm bead, we can visually estimate the right focus at different positions, and this has an effect on OVD measurement. We can underestimate it if we are really out of focus, compared with the equatorial plane (for a sphere). We have shown that there is a sufficient range of several micrometers in $Z$ where OVD does not significantly change, either for a $40-\mathrm{mm}$ mitotic cell in culture medium or for a $30-\mathrm{mm}$ polystyrene bead in immersion oil. In these conditions, OVD of the bead is 7 times as high as than OVD of the cell and is still equal to the theoretical one. That means that we can measure the actual OVD and thus the right dry mass of bigger cells, may be even of many layers of cells, with our simple imaging conditions. This is something that needs to be further investigated. The effect of spatial illumination coherence on OVD has also been studied for two objectives, for an adherent cell 
and for a $30 \mu \mathrm{m}$ polystyrene bead. With $40 \times$ NA 0.75 , the dry mass of a cell does not change much with the opening of the aperture diaphragm, till half of the maximum aperture of the condenser. With $100 \times$ NA 1.3 , the OVD of the bead does not variate by $>2 \%$ even at full aperture when compared with the minimal aperture (5\% of total aperture). This means that even for a big object with an OVD up to 7 times the OVD of a mitotic cell, we can use spatially incoherent illumination at high magnification. It is, however, recommended to work with low-coherence illumination for dry mass measurement. The insensitivity to illumination coherence makes the process independent on the operator who can accidentally change the aperture diaphragm setup.

In case of high throughput studies where thousands of cells are imaged, low magnification can be requested. For large OPD cells (mitotic, colonies), the illumination coherence control becomes important to correctly recover the cell OPD map. For magnification lower than $10 \times$, it is recommended to use laser illumination, which ensures the largest coherence possible. Otherwise artifacts appear which makes the dry mass values unstable and false. As an example, the technique was applied to quantify red blood cells dry mass of an entire population giving results in total accordance with literature values.

\section{Appendix: Segmentation Algorithm}

The segmentation process can be split in two steps: a first step to separate the pixels corresponding to cells from the pixels of the background and a second step to determine the number of cells and their contour.

Step 1: Separation of cells from the background. As the background is not constant but presents slow spatial variations, the background has first to be estimated before detecting the cells. For that purpose, the norm of the image gradient is calculated to reveal the presence of cell materials [see Fig. 14(b)]. An automatic thresholding is performed on this gradient image (the statistics of the gradient in background areas having been learned previously on a free-cell image), as shown in Fig. 14(c). To avoid the presence of cell pixels in the background area, a morphologic dilatation is then employed [see Fig. 14(d)]. The background can now be estimated as a 2-D polynomial function with order $d$ (typically $d=8$ in these experiments), using only the pixels gray levels in this background area [see Fig. 15(d)]: the corresponding estimated background is shown in Fig. 15(c). This background can finally be subtracted from the OPD image [see Fig. 15(b)], allowing separating the pixels of the cells from the background pixels using a simple thresholding of this image, the threshold being directly deduced from the background statistics.

Step 2: Determination of cell number and contours. In this step, only the pixels detected as cells are considered, the other being masked. For eukaryotic cells, the local maxima of OPD values are used to detect the number and approximate location of every cell. More precisely, in order to be robust to noise and to the presence of small materials inside the cells that can lead to an over estimation of the number of cells, the OPD image is first averaged (using a Gaussian kernel for which size has to be fixed by the user) before searching for the local maxima [see Fig. 16(a)]. Since the size of the Gaussian kernel is linked to the minimal size of the cells that are analyzed, this parameter allows the user to give an a priori knowledge on the minimal (a)

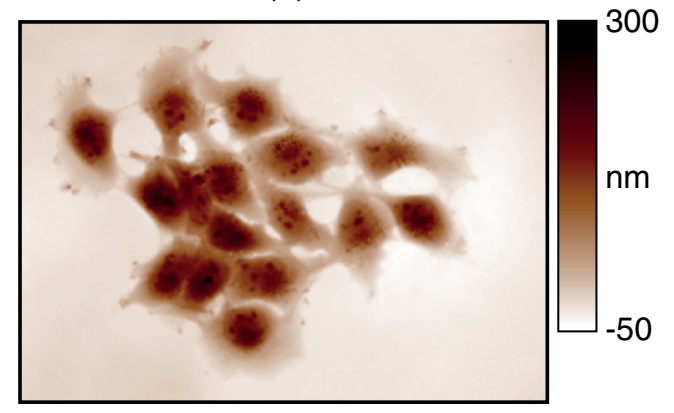

(c)

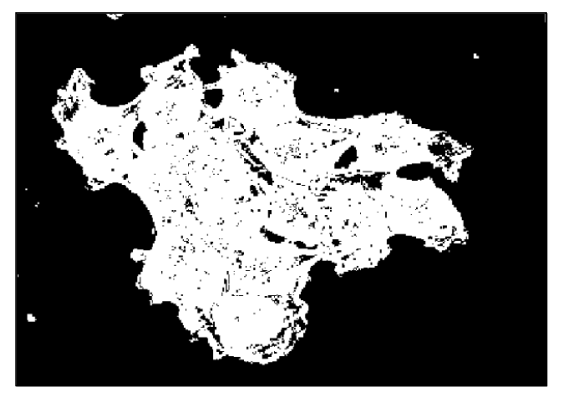

(b)

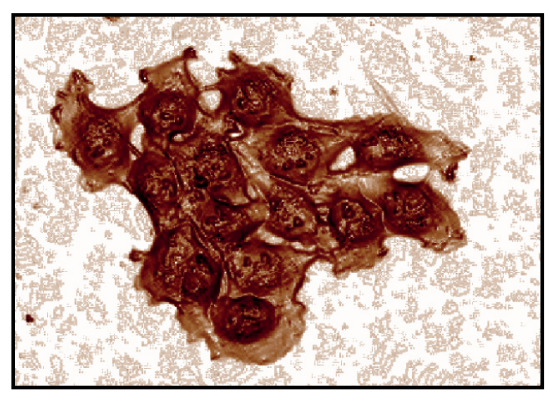

(d)

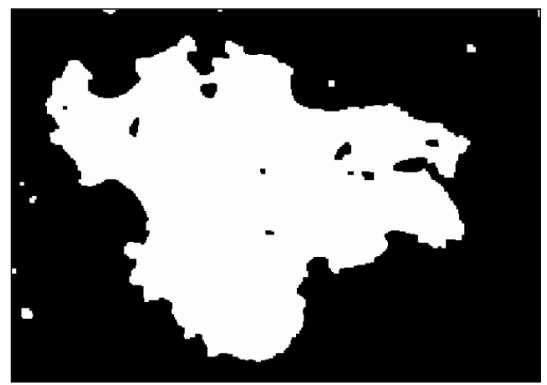

Fig. 14 Example of automatized segmentation on COS-7 cells imaged with a 40x magnification, $N A=1.3$. (a) Raw OPD image, (b) OPD gradient norm (in log scale for visual purpose), (c) automatic threshold, and (d) dilatation of image (c). 
(a)

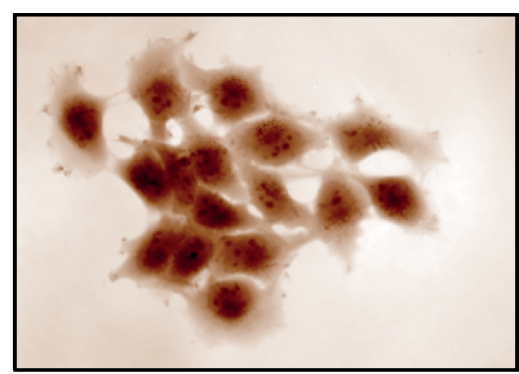

(c)

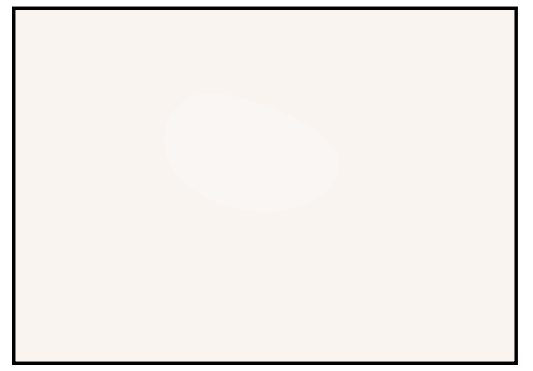

(b)

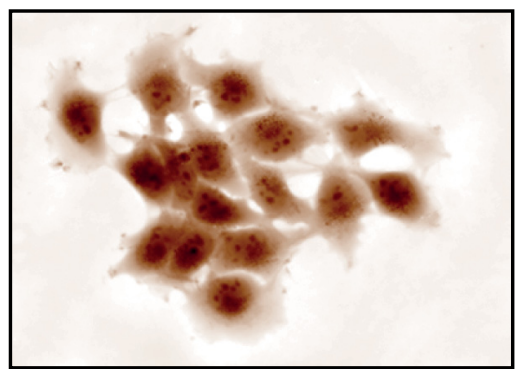

(d)

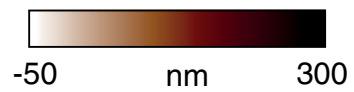

Fig. 15 Example of automatized segmentation on COS-7 cells imaged with a $40 \times$ magnification, $N A=1.3$. (a) OPD raw image, (b) OPD with flattened background, (c) evaluated background thanks to a projection on an eight-order two-dimensional (2-D) polynomial basis, and (d) background visualization.

(a)

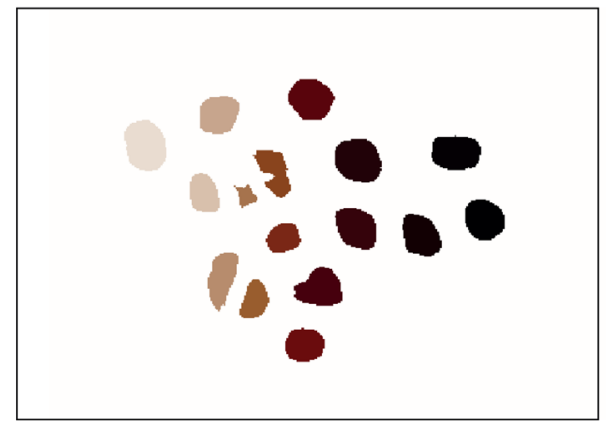

(c)

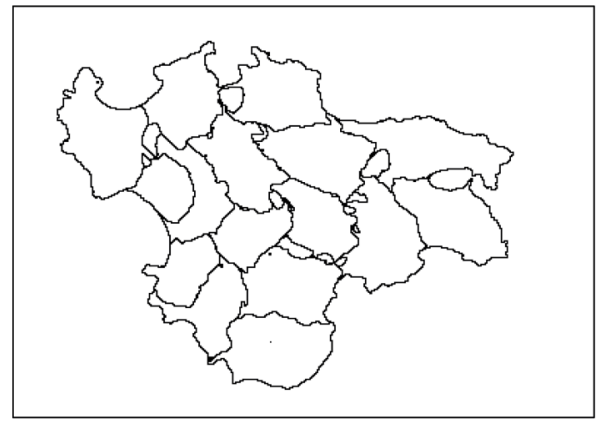

(b)

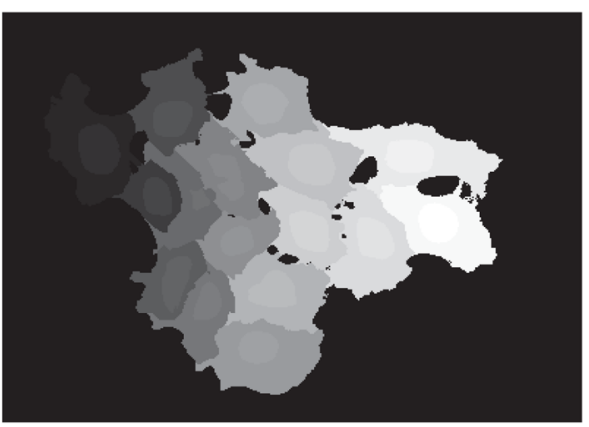

(d)

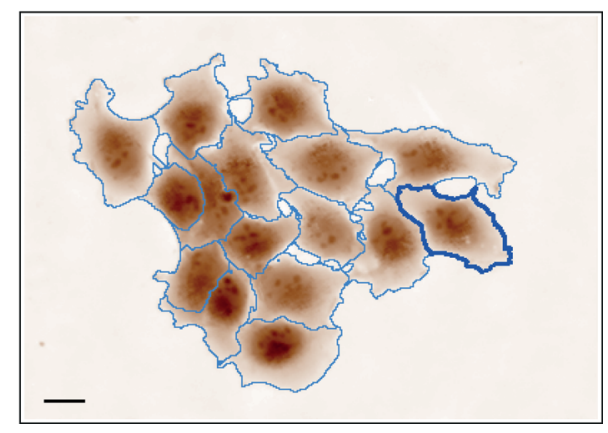

Fig. 16 Example of automated segmentation on COS-7 cells imaged with a $40 \times$ magnification, NA = 1.3. (a) Detected nuclei, (b) detected cells, (c) cells, and (d) final segmentation results. 
size of the cells that have to be recovered. The precise contours of the cells are then deduced from these initial seeds using a watershed algorithm [see Fig. 16(b), the corresponding contours being shown in Figs. 16(c) and $16(\mathrm{~d})]$.

A problem can come from the fact that if a cell is partly in and out of the field of view, with its nucleus outside, and touches the one you want to study, part of this cell can be fused with the other, leading to overestimated surface and dry mass. Segmentation has to be visually checked and in this case manually corrected. Another issue comes for cells superimposition, where OPD on several pixels is due to two or more cells. We cannot determine which part of the OPD comes from one cell, but no other technique can neither.

\section{Acknowledgments}

Financial support from the QuITO project funded by Fonds Unique Interministériel and Provence Alpes Cote d'Azur Region is gratefully acknowledged. We acknowledge financial support from France Bio-Imaging (ANR Grant InfrastructureNo. ANR-10-INBS-04-01). Conflict of interest: The authors declare the following competing financial interest: PHASICS Company is the supplier of the QWLSI system that is the key element of the technique described in this paper.

\section{References}

1. R. Benjamin et al., "Noninvasive characterization of the fission yeast cell cycle by monitoring dry mass with digital holographic microscopy," J. Biomed. Opt. 14, 034049 (2009).

2. P. Girshovitz and N. T. Shaked, "Generalized cell morphological parameters based on interferometric phase microscopy and their application to cell life cycle characterization," Biomed. Opt. Express 3(8), 1757-1773 (2012).

3. K. L. Cooper et al., "Multiple phases of chondrocyte enlargement underlie differences in skeletal proportions," Nature 495(7441), 375-378 (2013).

4. T. A. Zangle et al., "Quantifying biomass changes of single CD8+ t cells during antigen specific cytotoxicity," PLoS One 8(7), e68916 (2013).

5. T. A. Zangle et al., "Quantification of biomass and cell motion in human pluripotent stem cell colonies," Biophys. J. 105(3), 593-601 (2013).

6. Y. K. Park et al., "Spectroscopic phase microscopy for quantifying hemoglobin concentrations in intact red blood cells," Opt. Lett. 34(23), 3668-3670 (2009).

7. T. A. Zangle and M. A. Teitell, "Live-cell mass profiling: an emerging approach in quantitative biophysics," Nat. Methods 11(12), 1221-1228 (2014).

8. G. Popescu et al., "New technologies for measuring single cell mass," Lab Chip 14(4), 646-652 (2014).

9. S. Di Talia et al., "The effects of molecular noise and size control on variability in the budding yeast cell cycle," Nature 448, 947-951 (2007).

10. M. Godin et al., "Using buoyant mass to measure the growth of single cells," Nat. Methods 7, 387-390 (2010).

11. S. Son et al., "Direct observation of mammalian cell growth and size regulation," Nat. Methods 9(9), 910-912 (2012).

12. F. F. Delgado et al., "Intracellular water exchange for measuring the dry mass, water mass and changes in chemical composition of living cells," PLoS One 8, e67590 (2013).

13. K. Park et al., "'Living cantilever arrays' for characterization of mass of single live cells in fluids," Lab Chip 8(7), 1034-1041 (2008).
14. R. Barer, "Interference microscopy and mass determination," Nature 169(4296), 366-367 (1952).

15. G. Popescu et al., "Erythrocyte structure and dynamics quantified by Hilbert phase," J. Biomed. Opt. 10, 060503 (2005).

16. G. Popescu et al., "Optical imaging of cell mass and growth dynamics," Am. J. Physiol.: Cell Physiol. 295, C538-C544 (2008).

17. S. Yongjin et al., "Size homeostasis in adherent cells studied by synthetic phase microscopy," Proc. Natl. Acad. Sci. U. S. A. 110(41), 16687-16692 (2013).

18. M. Mir et al., "Optical measurement of cycle-dependent cell growth," Proc. Natl. Acad. Sci. U. S. A. 108, 13124-13129 (2011).

19. Z. Wang et al., "Spatial light interference microscopy (SLIM)," Opt. Express 19(2), 1016-1026 (2011).

20. K. G. Phillips, S. L. Jacques, and O. J. T. McCarty, "Measurement of single cell refractive index, dry mass, volume, and density using a transillumination microscope," Phys. Rev. Lett. 109, 118105 (2012).

21. P. Bon et al., "Quadriwave lateral shearing interferometry for quantitative phase microscopy of living cells," Opt. Express 17(15), 1308013094 (2009).

22. P. Bon et al., "Enhanced 3D spatial resolution in quantitative phase microscopy using spatially incoherent illumination," Opt. Express 22(7), 8654-8671 (2014).

23. P. Bon et al., "Optical detection and measurement of living cell morphometric features with single-shot quantitative phase microscopy," J. Biomed. Opt. 17(7), 076004 (2012).

24. P. Bon et al., "Fast label-free cytoskeletal network imaging in living mammalian cells," Biophys. J. 106, 1588-1595 (2014).

25. J. Primot and N. Guérineau, "Extended Hartmann test based on the pseudoguiding property of a Hartmann mask completed by a phase chessboard," Appl. Opt. 39(31), 5715-5720 (2000).

26. P. Bon, S. Monneret, and B. Wattellier, "Noniterative boundary-artifactfree wavefront reconstruction from its derivatives," Appl. Opt. 51(23), 5698-5704 (2012).

27. S. Aknoun et al., "Quantitative retardance imaging of biological samples using quadriwave lateral shearing interferometry," Opt. Express 23, 16383-16406 (2015).

28. S. Bernet et al., "Quantitative imaging of complex samples by spiral phase contrast microscopy," Opt. Express 14(9), 3792-3805 (2006).

29. E. Cuche, F. Bevilacqua, and C. Depeursinge, "Digital holography for quantitative phase-contrast imaging," Opt. Lett. 24(5), 291-293 (1999).

30. B. Kemper and G. von Bally, "Digital holographic microscopy for live cell applications and technical inspection," Appl. Opt. 47(4), A52-A61 (2008).

31. G. Popescu et al., "Fourier phase microscopy for investigation of biological structures and dynamics," Opt. Lett. 29(21), 2503-2505 (2004).

32. H. Sierra, C. A. DiMarzio, and D. H. Brooks, "Modeling phase microscopy of transparent three-dimensional objects: a product-of-convolutions approach," J. Opt. Soc. Am. A 26(5), 1268-1276 (2009).

33. P. Bon, B. Wattellier, and S. Monneret, "Modeling quantitative phase image formation under tilted illuminations," Opt. Lett. 37(10), 17181720 (2012).

34. H. B. Mann and D. R. Whitney, "On a test of whether one of two random variables is stochastically larger than the other," Ann. Math. Stat. 18, 50-60 (1947).

35. T. S. Ralston et al., "Inverse scattering for optical coherence tomography," J. Opt. Soc. Am. A 23(5), 1027-1037 (2006).

36. L. E. H. Whitby and C. J. C. Britton, "Disorders of the blood," in Diagnosis: Pathology: Treatment: Technique, xii +759 pp., Churchill, London (1950).

37. K. G. Phillips, S. L. Jacques, and O. J. T. McCarty, "Measurement of single cell refractive index, dry mass, volume, and density using a transillumination microscope," Phys. Rev. Lett. 109(11), 118105 (2012).

Biographies for the authors are not available. 